\title{
Erratum: Chromaticity of the lattice and beam stability in energy recovery linacs \\ [Phys. Rev. ST Accel. Beams 15, 074401 (2012)]
}

\author{
Vladimir N. Litvinenko \\ (Received 25 November 2014; published 31 March 2015)
}

DOI: 10.1103/PhysRevSTAB.18.039901

PACS numbers: 29.27.-a, 29.20.Ej, 99.10.Cd

The subject of emittance growth resulting from noncompensated chromaticity in single pass linac focusing systems was exhaustively studied in the era of linear colliders decades ago; see, for example, classical treatment in Refs. [1-4], and references therein. Effects described in these papers are fully applicable to energy recovery linacs with noncompensated chromaticity, e.g., a case presented in this paper. I suggest to use a strongly chromatic lattice to improve the beam's stability in energy recovery linacs (ERLs). Recently, I realized, that, naturally, such lattices would exhibit a very high sensitivity to orbital errors - the effect described in details in Refs. [1-4]. Such errors could, in turn, significantly increase the beam's emittance. Hence, this Erratum will address this shortcoming of this paper.

A simple case illustrating the importance of this shortcoming can be presented in a very simple form [5] using notations used in this paper. The orbit distortion caused by random field errors can be described as follows:

$$
\delta x(s, \delta)=w(s, \delta) \cdot \int_{0}^{s} \frac{e \delta B\left(s_{1}\right)}{p_{o} c(1+\delta)} \cdot \sin \left(\psi(s, \delta)-\psi\left(s_{1}, \delta\right)\right) \cdot w\left(s_{1}, \delta\right) d s_{1}
$$

wherein I used the definitions from Eq. (8) of this paper. The angle $\delta x^{\prime}(s, \delta)$ can be obtained readily simply by differentiating (1). In a periodic lattice comprising $N_{\text {cell }}$ identical cells, introducing an angular kicks $\theta_{k}$ would result in the following energy-dependent orbit at its exit:

$$
\delta x(s, \delta)=w_{N}\left(s_{N}, \delta\right) \cdot \sum_{k=1}^{N_{\text {cell }}} \theta_{k} \cdot w\left(s_{k}, \delta\right) \cdot \sin \left(\psi\left(s_{N}, \delta\right)-\psi\left(s_{k}, \delta\right)\right) .
$$

For the case of uncorrelated errors, $\left\langle\theta_{i} \theta_{k}\right\rangle=\theta_{i}^{2} \delta_{i}^{k}$, located at the cells' centers, with a Gaussian energy distribution (14) and expansion (10), one can show [5] that the projected normalized emittance at the exit of the lattice can be written as follows:

$$
\begin{aligned}
\varepsilon_{N}^{2} & =\left(\varepsilon_{\mathrm{No}}+\gamma_{o} \beta \sum_{n=1}^{N_{\text {cell }}} A_{n} \theta_{n}^{2}\right)\left(\varepsilon_{\mathrm{No}}+\gamma_{o} \beta \sum_{n=1}^{N_{\text {cell }}} B_{n} \theta_{n}^{2}\right)+O\left(\left(v \sigma_{\delta} \sum_{n=1}^{N_{\text {cell }}} \theta_{n}^{2}\right)^{2}\right) \\
\beta & \equiv w^{2} ; \quad E_{n}=\exp \left(-\left(2 \pi n \cdot C \sigma_{\delta}\right)^{2}\right) ; \\
A_{n} & =\frac{1}{2}\left(1-E_{n}\right)\left(1-E_{n} \cos \left(2 n \mu_{o}\right)\right) ; \\
B_{n} & =\frac{1}{2}\left(1-E_{n}\right)\left(1+E_{n} \cos \left(2 n \mu_{o}\right)\right),
\end{aligned}
$$

where $\varepsilon_{\text {No }}$ is the beam's initial normalized emittance, $\gamma_{o}=E_{o} / m c^{2}$ is the beam's relativistic factor, $\mu_{o}$ is the phase-advance per cell, and $C$ is the cell's chromaticity. Again, for brevity, I discuss here only the main terms in (3) and neglect the small term $O(\ldots)$ [5].

I note that for an achromatic lattice $(C=0)$ or for a monoenergetic beam $\left(\sigma_{\delta}=0\right)$ there is no growth in emittance. In contrast, for a lattice dominated by chromaticity with $E_{n}=\exp \left(-\left(2 \pi n \cdot C \sigma_{\delta}\right)^{2}\right) \rightarrow 0$, which correspond to the "loss of memory," we can estimate the normalized emittance as follows:

Published by the American Physical Society under the terms of the Creative Commons Attribution 3.0 License. Further distribution of this work must maintain attribution to the author(s) and the published articles title, journal citation, and DOI.

1098-4402/15/18(3)/039901(2) 


$$
\Delta \varepsilon_{N}=\varepsilon_{N}-\varepsilon_{\text {No }} \sim N_{\text {cell }} \frac{\gamma_{o} \beta}{2}\left\langle\theta^{2}\right\rangle .
$$

Thus, for a 5-GeV beam, $N_{\text {cell }}=500$, and $\beta \sim 2 m$, the rms angular kicks of $10^{-6}$ rad could engender a 10-mm mrad growth of normalized emittance. Such an increase would not acceptable for most ERL projects. Thus, unless a remedy is found to prevent the growth of emittance, a careful study will be required to determine whether the proposed Transverse Beam Break-Up instability-suppression scheme would be acceptable for a specific ERL project.

Furthermore, following modifying Eq. (34) in Ref. [3] for the case of the constant energy, one can arrive to the simple equation of the emittance delusion caused by a corrected orbit in periodic FODO lattice (see [1-3] for details on the orbit correction assumptions):

$$
\Delta \varepsilon_{N} \approx 32 \pi \cdot \gamma_{o} \cdot N_{\text {cell }} \cdot C \frac{\left\langle\delta x_{\mathrm{BPM}}^{2}\right\rangle \sigma_{\delta}^{2}}{L_{\text {cell }}},
$$

where $\sqrt{\left\langle\delta x_{\mathrm{BPM}}^{2}\right\rangle}$ is an rms beam position monitor positioning error. For the above parameters and the beam with the energy spread of $0.03 \%$, the chromaticity per cell $\sim 0.25$, rms beam position monitor position errors $\sim 0.2 \mathrm{~mm}$, and cell length of $2 \mathrm{~m}$, one should expect the emittance growth $\sim 0.5 \mathrm{~mm}$ mrad.

Accordingly, the conclusion to this paper should be modified by adding the following:

The high chromaticity of the ERL's lattice, in combination with a sufficient energy spread that suppresses the response to the transverse kicks in the ERL's linac, also would result in a high sensitivity to random and correlated orbit errors. The latter, in turn, could engender a significant (at least for some applications) growth of emittance. Thus, the applicability of my proposed scheme to ERLs operating with extremely low emittance beams could be a challenging problem.

\section{ACKNOWLEDGMENTS}

The author thanks the reviewer of this Erratum for pointing out the key publications fully describing the emittance delusion in linear accelerators.

[1] Ronald D. Ruth, Frontiers of particle beams, Lect. Notes Phys. 296, 440 (1988).

[2] T. Raubenheimer, PhD thesis, Stanford University, SLAC-Report-387, 1991.

[3] T. Raubenheimer, Estimates of emittance dilution and stability in high-energy linear accelerators, Phys. Rev. ST Accel. Beams 3, 121002 (2000).

[4] M. Syphers, Report No. FN-458, 1987.

[5] V. N. Litvinenko, Chromatic lattices, orbit errors and emittance growth, C-AD note, BNL, 2014. 